\title{
Structure Familiale Et Disqualification De La Personne Agee Diabetique Residant A Micao Dans La Commune De Yopougon (Cote d'Ivoire)
}

\author{
Droh Antoine, \\ Enseignant Chercheur, \\ Institut National de la Jeunesse et des Sports (INJS) Abidjan, Cote d'Ivoire \\ Coulibaly Zoumana, \\ Enseignant-Chercheur, \\ Université Peleforo Gon Coulibaly de Korhogo, Cote d'Ivoire
}

Doi:10.19044/esj.2019.v15n34p175 URL:http://dx.doi.org/10.19044/esj.2019.v15n34p175

\section{Résumé}

L'objectif de la présente étude est d'analyser le rapport entre la structure familiale et la disqualification des personnes diabétiques âgées de 60 à 82 ans dans la commune de Yopougon. Elle aborde la question par une collecte de données qualitatives auprès des malades âgés diabétiques et de leurs aidants familiaux pour apprécier l'organisation et le fonctionnement de la cellule familiale, les représentations sociales du grand âge et du diabète, le vécu du sujet âgé face aux construits sociaux de l'entourage, et les stratégies plausibles dans le sens de l'amélioration des conditions de vie des aînés. Le rapport entre la structure familiale et la disqualification des personnes âgée diabétiques a été apprécié à travers une analyse de contenu. Les résultats ont montré une transformation du rôle et du statut des personnes âgées qui est consécutive aux mutations sociales. Ils mettent en lumière une distension considérable des liens affectifs et des rapports intergénérationnels au sein de la famille, de même qu'une situation de précarité et de vulnérabilité des séniors diabétiques. Leurs conditions de vie requièrent l'implication des élus locaux et l'urgence de la mise en œuvre d'une politique de prise en charge.

Mots clés : Côte d'Ivoire, Diabète, Disqualification, Personnes Agées, Structure Familiale 


\title{
Family Structures and Disqualification of Elderly Diabetics Residing in Micao in the Municipality of Yopougon (Ivory Cost)
}

\author{
Droh Antoine, \\ Enseignant Chercheur, \\ Institut National de la Jeunesse et des Sports (INJS) Abidjan, Cote d'Ivoire \\ Coulibaly Zoumana, \\ Enseignant-Chercheur, \\ Université Peleforo Gon Coulibaly de Korhogo, Cote d'Ivoire
}

\begin{abstract}
The aim of this study is to analyze the relationship between family structure and the disqualification of people with diabetes aged 60 to 82 in Yopougon commune. She addresses the issue by collecting qualitative data from elderly diabetic patients and their caregivers to appreciate the organization and functioning of the family unit, the social representations of old age and diabetes, the experience of the elderly subject in the face of social constructs, and plausible strategies to improve the living conditions of seniors. The relationship between family structure and the disqualification of elderly diabetics was appreciated through a content analysis. The results showed a transformation of the role and status of the elderly that follows social change. They highlight a considerable distension of emotional ties and intergenerational relationships within the family, as well as a situation of precariousness and vulnerability of seniors with diabetes. Their living conditions require the involvement of local elected officials and the urgency of implementing a care policy.
\end{abstract}

Keywords: Diabetes, Disqualification, Family Structure, Elderly People, Ivory Coast

\section{Introduction}

L'élévation de l'espérance de vie du fait des progrès scientifiques entraînerait actuellement l'augmentation de l'effectif des personnes âgées dans le monde. Ce phénomène démographique s'accompagne d'un ensemble de risques multiformes parmi lesquels figurent en bonne place les pathologies chroniques entraînant le plus souvent l'isolement social. Ce processus qui 
conduit à la mise à l'écart de ces personnes du troisième âge renvoie à ce que l'on appelle sous le vocable de disqualification de la personne âgée.

La disqualification se réfère à un procédé psychologique qui consiste à déclarer une personne inapte à faire quelque chose, à penser à propos d'un sujet etc. ou à faire croire qu'elle ne peut pas le faire ou que son avis ne doit pas être pris en compte. Elle peut avoir pour but de détruire la confiance en soi de la personne. C'est le cas des personnes âgées diabétiques. Pour ces personnes, la disqualification sociale renvoie au processus d'affaiblissement ou de rupture des liens du sujet diabétique avec son entourage (Paugam, 1991).

En effet, les maladies chroniques dont le diabète représentent actuellement un véritable fléau social dans le monde. L'Organisation Mondiale de la Santé (OMS), dans son premier rapport sur la question, affirmait que 422 millions de personnes dans le monde vivent avec cette maladie (OMS, 2016). Dans certains pays où la plupart des habitants ont des revenus faibles les conséquences socio-économiques du diabète semblent considérables. Ainsi l'on estime que, le coût du traitement représente 70 à $96 \%$ du budget familial pour les patients les plus pauvres et de 25 à $55 \%$ lorsque ceux-ci ont des revenus moyens (Amos, Yves et al, 2009). A cela, s'ajoute aussi le fait que le nombre de personnes atteintes de diabète suit la même courbe ascendante que celle se rapportant au nombre de personnes âgées (60 ans ou plus) dans le monde, depuis la fin du $\mathrm{XX}^{\text {ème }}$ siècle jusqu'à ce jour. Plus fondamentalement, entre 2000 et 2050, les experts estiment que le taux de seniors passera du simple au double $(11 \%$ à $22 \%)$ et qu'en nombre absolu, cette catégorie spécifique passera de 605 millions à 2 milliards (OMS, op. cit). Mais dans cette même frange de la population, la Fédération Internationale du Diabète (FID) estime que le taux de prévalence mondiale du diabète s'établit aujourd'hui à 18,6\% , soit 134,6 millions de personnes âgées souffrant de cette pathologie chronique et qu'à l'horizon 2035, ce nombre devrait passer à 252,8 millions (FID, 2013).

Face à ces projections, les pays africains qui peinent encore à mettre en place un système efficace de prise en charge des personnes âgées, de surcroît souffrant de maladies chroniques comme le diabète, n'ont de recours que la solidarité familiale (Loriaux, 2002). La Côte d'Ivoire, avec son système actuel de protection juridique et sociale en faveur des aînés, ne fait pas exception. Au mieux, l'on préfère dans cet espace territorial recourir aux solidarités privées intergénérationnelles pour assurer l'entretien des personnes âgées qui avaient d'ailleurs elles-mêmes misées sur leurs progénitures pour assurer la tranquillité de leurs vieux jours.

Mais aujourd'hui, la situation est toute autre, étant donné la fascination du modèle civilisationnel occidental sur la société ivoirienne, surtout en milieu urbain. Dans la commune de Yopougon, précisément à MICAO, les personnes âgées sont diabétiques en majorité et méconnues des services médicaux de 
prise en charge. Et aussi paradoxal que cela puisse paraître, le milieu familial qui devrait agir comme une institution de soutien et d'accompagnement social dans un contexte africain, génère une tension permanente qui impacte négativement la santé psychologique des sujets âgés. Ainsi, contribue-t-il à désagréger leur statut. Par ailleurs, ces derniers vivent, dans l'indifférence totale, du fait de l'affaiblissement des liens familiaux de solidarité. Ce qui constitue un facteur accentuant leur isolement et hypothéquant le pronostic de leur état pathologique.

Eu égard à ces transformations inattendues, l'objectif de la présente étude est donc d'analyser le rapport entre la structure familiale et la disqualification des personnes diabétiques âgées de 60 à 82 ans dans la commune de yopougon

\section{Méthodologique}

Il s'agit d'une étude de type transversale, à visée descriptive et analytique. Elle a débuté le 1er mars 2018 pour prendre fin le 31 juillet 2018. Elle a ciblé 60 Familles dont 51 seulement ont accepté de répondre aux questions. Au plan méthodologique, l'enquête de terrain a été essentiellement qualitative. L'étude s'est déroulée à MICAO dans la commune de Yopougon située dans le district d'Abidjan en Côte d'Ivoire. Les outils utilisés sont une grille d'observation, un guide d'entretien, un test d'évaluation de mémoire (cinq mots de DUBOIS) et le glucomètre pour mesurer la glycémie. L'enquête a été exhaustive car elle a concerné toutes les personnes âgées diabétiques visitées à domicile et qui ont accepté de participer à l'étude. Les personnes âgées non diabétiques ont été systématiquement exclues. Le recueil des données a été réalisé avec l'aide du chef de quartier et du médecin chef du centre de secours médical Bethesda pour la confirmation du diagnostic des sujets ignorant leur statut de diabétique. L'analyse de contenu nous a permis de faire ressortir l'organisation et le fonctionnement de la structure familiale ainsi que le vécu quotidien des enquêtés. Cette étude repose sur la théorie de la disqualification sociale (Paugam, 1991) qui renvoie au processus d'affaiblissement ou de rupture des liens du sujet diabétique avec son entourage.

\section{Résultats}

\section{II.1. Organisation et fonctionnement de la structure familiale des enquêtés}

L'âge des enquêtés variait entre 60 ans et 82 ans. Moins de la moitié des répondants est propriétaire d'un logement. 57\% sont locataires et seulement $6 \%$ habitent chez leurs enfants. Ils sont pour la plupart sans revenu fixe et vivent de soutien financier ou de don de personnes de bonne volonté. Pour ceux qui ont exercé une activité professionnelle avant leur départ à la 
retraite, ils jugent leur pension dérisoire, ne leur permettant pas de se prendre en charge convenablement. Toute chose qui fait de ces sujets, des personnes nécessiteuses dont l'accessibilité aux soins médicaux demeure problématique. Aucun ne bénéficie ni d'une assurance maladie, ni d'une sécurité sociale. Par ailleurs, $74 \%$ d'entre elles vivaient sous le seuil de pauvreté avec des petits enfants à leur charge. Parmi les répondants, 12 étaient diabétiques non connus et 39 étaient diabétiques connus, desquels $15 \%$ mettent sérieusement en doute le diagnostic médical. Ces derniers ne suivaient donc pas le traitement au motif que l'étiologie de leur mal est d'origine mystique comme en témoigne ce qui suit :

"Les médecins affirment que je souffre de diabète. Mais je ne crois pas à cela. Cette maladie n'est pas une maladie simple, c'est un sort, c'est une affaire de sorcellerie ». (Monsieur K., 68 ans).

Dans cette logique une veuve de 63 ans s'exprimant, lie l'origine de sa maladie aux biens matériels que son mari défunt lui a légués. Ce qui, selon elle, a suscité la haine de ses beaux-parents qui en veulent à sa vie.

"Ce n'est pas étonnant, dit-elle, ces maux dont je souffle ne sont rien d'autres que le résultat de la méchanceté de ma belle-famille. Tout simplement à cause des biens que le père de mes enfants a laissés après sa mort ». Ainsi, poursuit-elle : "ces sorciers aiment tout ce qui est matériel ou financier, la soif $d u$ gain constitue un motif de jalousie pour exercer leurs pratiques sorcières ».

A travers ces discours, l'on peut noter l'importance des représentations culturelles des enquêtés face à l'étiologie du diabète, notamment leurs croyances à la sorcellerie. L'approche psychodynamique de ces propos, montre que les répondants sont convaincus d'être la victime de l'ensorcellement par leurs proches. Aussi, le recours au service des guérisseurs traditionnels constitue-t-il le choix privilégié dans leur recherche de soins. Mais ce recours à la consultation des tradipraticiens est vivement contesté par leurs enfants qui voient en cela un gaspillage des ressources déjà limitées. Cette situation faite de suspicion, d'incompréhension, de vengeance crée un climat social d'insécurité et de méfiance qui désorganise le fonctionnement de la cellule familiale.

A côté de cela, nos résultats mettent en évidence que les personnes âgées, qui autrefois étaient considérées comme des vecteurs de socialisation, des guides et la mémoire de la famille, sont contestées dans leurs rôles d'antan. Elles sont souvent accusées d'être des sorcières. Alors, l'espace collectif, autrefois épanouissant pour tous les membres du lignage, voire de la communauté, tend-t-il à devenir un milieu vacant, sans maître, du fait d'une disqualification sociale ou d'une rupture d'équilibre pour la personne âgée diabétique. Plus de la moitié des répondants affirment clairement cet état d'affaiblissement de position sociale en ces mots : 
"Je ne suis plus consulté dans la prise des décisions. Même si cela arrive, mes avis ne sont pas pris en compte. Je ne sais pourquoi ? Est-ce parce que je n'ai plus ni force ni moyen? ou c'est parce que je suis malade?" S'interroge ainsi T.R âgé de $\mathbf{7 7}$ ans, ancien ouvrier de l'usine MICAO.

La dégradation des conditions de vie et du niveau des rapports étaient visibles. $37 \%$ des enquêtés le reconnaissent et disent qu'ils vivent une crise identitaire et d'affaiblissement des liens sociaux, depuis que leurs ressources financières sont épuisées, et surtout du fait de la précarité de leur état de santé. En outre, le regard quotidien chez les aidants familiaux interrogés est dominé par l'impression que leur parent âgé est de trop.

\section{II.2. Représentations sociales du grand âge et du diabète par la famille}

Dans l'entretien avec la famille, une question de vocabulaire apparait : « vieillesse, vieux, personnes âgées, retraités, seniors et 3e âge ». Les mots ne sont pas neutres. Ils traduisent et construisent des représentations imaginées par les aidants familiaux. Certaines représentations se fondent sur l'âge, d'autres s'élaborent sur l'attitude plus ou moins optimiste face à la vie, aux métaphores traditionnelles faisant de la vieillesse l'arrière-saison de la vie, le couchant ou le soir, se substituent des images plus qu'inquiétantes, comme « la fin du parcours ». Cette question de vocabulaire est l'expression vivace que les membres de notre population d'étude vivent dans une société qui véhicule des croyances, des normes et des valeurs, face auxquelles ils construisent des représentations sociales sur des objets sociaux. Ainsi, les questions sur les représentations sociales du grand âge et du diabète dévoilent qu'il existe une pensée «profane » sur les deux concepts (diabète et vieillissement). Le diabète est vécu par les sujets âgés comme une expérience individuelle comportant des retentissements psychologiques, sociaux et culturels. Alors que la famille l'aborde comme une charge symbolique pour l'ensemble du groupe social. Par ailleurs, le vieillissement est représenté à la fois comme une charge socioéconomique, un fardeau et une menace puisque le vieillard est soupçonné d'être le sorcier de la famille, donc responsable du chômage des jeunes et une barrière aux mariages des jeunes filles. Les propos qui suivent étayent mieux les représentations que les familles se font du grand âge et de la pathologie des parents âgés.

D.Y : «Avoir à sa charge une maman âgée est difficile; et de surcroit malade, c'est très pénible. Les médecins nous disent que le diabète est une maladie incurable causée par la consommation excessive des aliments sucrés. C'est vraiment lourd sur le plan financier mais aussi voir sa génitrice devenir subitement triste, soucieuse avec des sauts d'humeur et parfois des problèmes de vue et d'audition, euh, euh! C'est dur. »

La réponse de G.L sur la question est différente. Pour lui les personnes âgées en général sont malades à cause des mauvaises actions qu'elles ont 
posées : «Ce que je sais, c'est la vieillesse. En dehors de ça, je ne sais pas ce dont souffre le vieux. Lui, il a fait son temps, au lieu de partir, il bloque la bénédiction de ses enfants. Je ne travaille pas, mes deux sœurs aînées ne sont pas dans un foyer. Seul mon petit frère qui a eu la chance de s'échapper de la sorcellerie du vieux pour aller en Italie a pu gagner sa vie. Dans la journée, le vieux fait semblant de somnoler, et la nuit, il veille. Pourquoi ? Ce n'est pas de la sorcellerie ça!»

Cet enquêté, âgé de 33 ans, ignore la maladie dont souffre son père et l'assimile au vieillissement. Dans ses propos, il considère le grand âge comme la finition, et explique que durer en vie est synonyme d'être responsable des malheurs de sa progéniture. Cependant, l'intervention de B.S, un autre répondant, fait ressortir le contraire :

«Notre papa est diabétique connu depuis cinq ans. Les causes réelles, nous les ignorons. Nous faisons ce que nous pouvons parce que la prise en charge demande beaucoup de moyens. Or, l'Etat n'a rien prévu pour le grand âge comme dans les pays développés. Notre éloignement des centres de diabétologie rend difficile l'accès aux soins ».

$\mathbf{Z}$. V, agent de la police nationale, ne dit pas le contraire. Pour lui, le grand âge est la preuve manifeste de la réussite du groupe :

"Atteindre le grand âge est considéré comme un bonheur dont on doit se réjouir; on ne peut pas, comme en Occident, mettre la personne âgée à l'écart dans une maison de retraite; dans une société de pure oralité, le vieux vit au milieu des siens, car il est le symbole de la continuité de la famille et la mémoire du groupe. C'est triste et regrettable qu'on les traite aujourd'hui de sorciers. »

Le recueil des informations montre que le vieillissement pathologique constitue une charge énorme pour la famille et l'image idyllique que la société ivoirienne se faisait du grand âge commence à battre de l'aile avec la nouvelle génération. L'ordre social ancien est en train d'être renversé. L'aîné, le vieux ou la vieille, gardien de l'expérience de la civilisation basée sur l'oralité, est en déclin, ne faisant plus le poids devant la modernité actuelle. Cependant, le regard des membres de la famille sur la vieillesse et le diabète bien qu'ambivalent n'exclut pas totalement l'importance accordé aux parents âgés. L'enquête révèle que le pouvoir gérontocratique est désormais démystifié et la tendance générale reste que le statut des séniors est confronté à un processus d'exclusion et de dégradation chez la totalité des enquêtés. C'est dans ce contexte que les personnes âgées interrogées nous expliquent leur vécu à travers ce qui suit. 


\section{II.3. Vécu du sujet âgé face aux construits sociaux de l'entourage sur le diabète}

La dégradation de l'état de santé affecte profondément la psychologie des personnes âgées interrogées. $81 \%$ d'entre elles, sans distinction de sexe ni de conditions sociales parlent de leur vécu quotidien comme suit : «je ne suis plus physiquement en forme ; j'ai du mal à accomplir certains travaux et à me déplacer comme auparavant, si bien que dans la journée je suis assis là comme ça», affirme d'une voix tremblante un retraité de MICAO assis isolé sur une chaise en bois. On note de leurs propos un vécu quotidien caractérisé par une disqualification du lien social qui se manifeste par une rupture d'équilibre interne et un isolement social. En effet, ce retraité âgé diabétique évoque une transformation physique et psychique qui crée en lui une angoisse permanente. Il a une mauvaise image de son schéma corporel qui entraîne une perte de l'harmonie interne. Ce dernier poursuit toujours avec une voie basse entrecoupée de pause : "Parce que je n'ai plus les moyens et que je suis fatigué par la maladie, au lieu de me venir en aide, on me traite de vieux sorcier. Quand j'entends souvent cela, j'éprouve un sentiment de déshonneur mais je n'ai pas les capacités de faire autrement ».

Cette étude montre que près de la moitié de ces malades âgés sont dans l'impossibilité d'améliorer leur sort du fait de la modification physiologique et psychologique. L'enquête montre un affaiblissement du rapport à l'entourage et une inexistence des aides sociales surtout que les personnes susceptibles de leur apporter un soutien sont elles-mêmes confrontées aux aléas du chômage. Un jeune ouvrier au chômage intervient en ces termes : "Quand je travaillais, j'achetais régulièrement l'insuline de ma mère. Depuis trois ans que je ne travaille pas, j'arrive difficilement à faire face aux dépenses de ma propre famille et à celles de mes parents. C'est dur! Mais c'est la réalité du pays. »

Un enquêté de 65 ans affirme ceci : «ma femme et moi, on ne s'entend pas très bien parce qu'elle est un peu autoritaire et elle m'empêche prendre un verre de Koutoukou dans le quartier ». Pour lui, tout est compliqué et sa femme est toujours occupée à ses activités commerciales. Ce qui fait qu'elle n'est pas souvent disponible pour lui.

Les contraintes du régime alimentaire, de la prise des médicaments et l'interdiction de la consommation de l'alcool et de certaines boissons gazeuses ont un impact sur le vécu des enquêtés. Les enquêtés expliquent se sentir disqualifiés socialement à partir du moment où ils sont maintenus, contre leur gré, dans une situation qui les prive de tout ou partie de leur dignité. Ils disent avoir le sentiment d'être " déclassées ", c'est-à-dire d'être dans une situation inférieure à celle qu'elles ont connue antérieurement. La maladie et la vieillesse sont vécues comme un déterminant social d'isolement chez $16 \%$ des enquêtés. L'étude fait ressortir le manque d'une politique véritable de prise en 
charge des personnes âgées. Ce qui les met dans la précarité et la vulnérabilité qui réduisent leur espoir de « vieillesse heureuse ».

L'ensemble des personnes âgées diabétiques interrogées sont affaiblis et sans ressources, ni sécurité sociale. Dans un environnement où le développement de l'individualisme et l'affaiblissement des liens classiques de solidarité familiale est inexistant, ils en appellent à la contribution des pouvoirs publics.

\section{II.4. Contribution à l'amélioration des conditions de vie des aînés en Côte d'Ivoire}

Les solidarités publiques et privées sont nécessaires là où la pauvreté réduit à la fois le pouvoir d'achat et l'accès aux services de santé des populations âgées. Dans l'entretien qui suit, 100\% des sujets âgés interrogés appellent l'aide du gouvernement alors qu'environ 30\% des aidants familiaux proposent que leurs parents âgés changent de comportement envers leurs proches. Cependant, une minorité non négligeable (40\%) estime que l'amélioration des conditions de vie des personnes âgées passe avant tout par l'amélioration des conditions existentielles de leurs progénitures. Ils estiment que le soutien familial, étain d'affects, reste pour le vieillard et ses proches le premier support et le dernier recours en cas de détresse humaine (maladie, décès, etc.). Pour une autre franche, environ 6\%, seule une meilleure condition socioéconomique des enfants améliore les rapports intergénérationnels et favorise l'entraide familiale.

Toute une panoplie de pistes pour contribuer à lutter à la fois contre la disqualification et le vieillissement pathologique des séniors a ainsi été exprimée de vive voix par la quasi-totalité de la population de notre étude.

Z. C, gendarme à la retraite, marié et père de 06 enfants dont 4 vivent encore sous le toit paternel, demande à l'Etat d'orienter les programmes de capitalisation aussi en faveur des chefs de ménage : «Les mesures préconisées par le gouvernement pour réduire la pauvreté privilégient les jeunes et les femmes, et n'accordent aucune place aux personnes du troisième âge. Or, le statut de chef de famille leur confère des responsabilités. Lorsque les programmes de développement mis en place par l'Etat les excluent, alors leur pouvoir s'affaiblit et la société les marginalise. " Il apparaît dans ces propos que les dépenses de l'Etat dans le social ne doivent pas être considérées comme un gaspillage mais comme un investissement dans le capital humain. Abondant dans ce même sens. D.T préconise donc l'implication de la municipalité et des conseils régionaux dans le médico-social: "Les collectivités territoriales doivent adopter une approche de protection sociale capable de répondre valablement aux besoins des personnes âgées. Elles doivent conjuguer les systèmes formels et non formels de sécurité sociale en reconnaissant qu'il existe aujourd'hui une proportion importante de 
personnes âgées qui n'a pas accès au système formel parce qu'elle n'a jamais eu accès à un emploi salarié ».

Contrairement à ce qui précède un nombre non négligeable de répondants mettent en exergue la nécessité de création d'emploi pour les jeunes afin de pouvoir s'occuper de leurs parents vieillissants. M.F déclare : «Le manque de revenus adéquats est le problème le plus crucial pour nous et nos parents âgés. Si vous n'avez pas de travail, de moyens, pas d'accès aux soins, pas de logement non plus, finalement, ce sont des palabres à en point finir dans la famille. »

Par contre, K. L prône, quant à elle, la formation des spécialistes en matière de prise en charge des vieux et la construction des structures médicosociales pour personnes âgées : "Le processus du vieillissement est complexe. Il faut spécialement former des gens à cela ; en plus, il faut créer des hôpitaux et services pour les vieux en Côte d'Ivoire. "

\section{Discussion}

L'analyser du rapport entre la structure familiale et la disqualification des personnes diabétiques âgées de 60 à 82 ans dans la commune de yopougon dans la présente étude met en évidence des facteurs susceptibles d'expliquer cette corrélation.

Les organisations familiales étendues en Afrique subsaharienne sont fondées sur divers modes de filiation et divers types de résidences, organisées autour de principes hiérarchiques définis selon le principe de séniorité (Barou, 2012). Mais selon l'auteur, ces organisations connaissent un bouleversement structural depuis la fin du XXe siècle avec l'exode rural, l'urbanisation et les crises politiques qui ont plutôt conduit à une diversification accrue des formes de familles.

Dans cet ordre d'idée, Neirynck et Melen (2016) témoignent que les systèmes familiaux sont en constante réorganisation. Toutefois, cette réorganisation est contenue par les mythes, les croyances et valeurs propres à chaque système; et si le vieillissement en lui-même entraîne déjà des adaptations dans l'organisation familiale, les maladies qui l'accompagnent ajoutent quelque chose de "catastrophique" qui va tantôt faciliter, tantôt freiner l'intégration et le soutien accordés à ses membres âgés. Ces deux auteurs estiment que l'être humain est tributaire des liens qu'il crée avec son environnement. Autrement dit, de l'attachement qu'on lui porte et qu'il porte aux siens dépend la condition de survie de l'espèce. La forme et la qualité des liens évoluent avec les cycles de la vie. La cellule familiale, creuset de cette construction relationnelle et identitaire, peut être havre de sécurité comme d'aliénation. Les personnes âgées, peuvent subir de rapides et nombreuses transformations, ajoutent-ils. Les résultats de notre travail mettent en évidence cette réalité. En effet, nos interviewés ont affirmé que le statut privilégié des 
vieillards leur conférait des rôles d'éclaireur de la famille au sein des sociétés traditionnelles. Mais le vieillissement, couplé avec les pathologies chroniques dont le diabète, sont désignés par ces derniers comme des marqueurs de perturbation du fonctionnement de la famille et de destruction de sa cohésion. Les familles se disloquent et les vieux sont victimes de maltraitance comme les travaux de Tanoh (2007) l'affirment en ces termes: «avec l'occidentalisation de la société ivoirienne par le biais de la colonisation, la famille de type nucléaire tend à se substituer à celle de type élargi, les personnes âgées ressentent une rupture entre elles et les autres membres de la famille. » Le constat d'une telle perturbation est que l'individu est dépouillé de la morale collective, la famille étant gravement affectée par les changements sociaux, économiques et démographiques. S'agissant des représentations sociales du grand âge et du diabète, les données recueillies auprès des membres de la famille des personnes âgées indiquent que le vieillard était autrefois entouré de prévenances. Ainsi, atteindre le grand âge était-il considéré comme un bonheur dont l'entourage se réjouissait. De son côté, Thomas (1983) fait relever ce prestige considérable dont jouissaient les vieux dans les vingt-deux ethnies qu'il a pu étudie sur le continent africain. Dans ces ethnies, l'image idyllique que l'africain se fait du vieillard est qualifiée par des expressions «Expérience, disponibilité, éloquence, savoir, sagesse ». Et ceci malgré la réalité des vieux séniles, égoïstes, tyranniques ou acariâtres. Ce rôle social, au départ si important, va sans cesse être remis en cause selon l'auteur au profit de la pénétration du livre et de l'écrit dans les sociétés africaines. Puisque l'acquisition des connaissances à travers les écritures et autres moyens de communication instruisent et éclairent les jeunes, alors le pouvoir des vieux est contesté, démystifié et même agressé. Ces conséquences mises en évidence dans le travail de Thomas sont comparables aux discours de certains de nos enquêtés qui trouvent que la présence du vieux est de trop.

Pour ce qui concerne la représentation dont la famille se fait du diabète, nos travaux révèlent que les sujets interviewés ont des conceptions plurielles de la pathologie. Celles-ci traduisent et influencent leur rapport à la maladie. De façon générale, l'étude affirme sans ambiguïté que le chômage des jeunes, la mauvaise alimentation liée à la précarisation de la situation socioéconomique des familles sont à la base du diabète. Ils ajoutent à cela la sédentarité et l'absence d'activité physique chez leurs vieux parents diabétiques. Cette vision qui résulte de notre enquête est superposables à celle de Poulain (1996) qui affirme que la misère, le taux de chômage élevé et les émeutes de faim engendrent l'émergence d'une obésité de précarisation. Ce qui entraîne selon Diabètes Leadership forum (2010), qu'en Afrique, le diabète touche de plus en plus les personnes démunies en milieu urbain. Poulain (op.cit) fait remarquer que cette obésité de précarisation associe une 
obésité de transition consécutive à des transformations alimentaires et à des manières de vivre, caractérisées principalement par la réduction de l'activité physique et la sédentarité. Cette obésité est souvent perçue par les communautés comme un symbole de bonne santé et d'opulence qui contraste avec l'état de santé du sujet. Dans ce cas la culture devient une variable étiologique en tant qu'elle peut conduire à la dégradation de l'état de santé par la production de présupposés qui favorisent l'installation du diabète. En outre, l'origine congénitale du diabète est mal connue des populations qui expliquent la résurgence de cette maladie à différents niveaux de l'arbre généalogique de certaines familles par la malédiction liée à la transgression d'un tabou.

Par ailleurs, cette maladie représente pour les membres de la famille un fardeau socio-économique aux conséquences difficiles à supportées. Ainsi, les personnes âgées qui devraient bénéficier de l'assistance et de la ferveur familiale se sentent victimisées, eu égard au coût de la prise en charge médicale et aux contraintes de leur régime alimentaire qui bouleverse les habitudes nutritionnelles de la famille. Il convient de reconnaître que le diabète tout comme les pathologies chroniques comporte des contraintes lourdes pour les patients et implique d'importantes conséquences financières au niveau familial. Ce problème est d'autant plus important dans la prise en charge du diabète que dans le rapport sur la santé, l'OMS affirme qu'« en absence de politiques efficaces pour créer des environnements propices à un mode de vie sain, et faute d'accès à des soins de santé de qualité le diabète non maitrisé a de graves conséquences pour la santé et le bien-être. Ces complications grèvent sérieusement les finances des patients et de leur famille, ainsi que l'économie des nations »(OMS, 2016). Le nombre d'études décrivant les causes possibles du diabète et ses conséquences au cours des vingt dernières années est important. Ces études confirment l'expressivité émotionnelle sur le vécu des sujets âgés diabétiques que nous avons visités.

Ainsi, la question du vécu de nos enquêtés face aux construits sociaux de l'entourage se résume en grande partie à la solitude ressentie ainsi que des souffrances physiques et morales qui en découlent. Ces résultats sont identiques aux écrits de Paillat qui relatent que: «Nul ne peut étudier la place des anciens dans notre société sans être conscient de la fréquence de leur isolement (au sens physique du terme) qui est facile à mesurer et de celle de la solitude (au sens affectif : se sentir seul) qui, elle, est difficile à saisir mais dont on pressent l'importance grâce aux confidences des enquêtés qui sont parfois moins réservés sur ce sujet qui leur tient à cour en face d'un enquêteur que vis-à-vis de leur propre famille pour peu que celle-ci, souvent pour de bonnes raisons, ne leur accorde pas l'attention, la sollicitude qu'ils en attendent. Que dire alors de ceux qui sont privés de famille et qui n'ont pas su ou pas pu créer un réseau amical de substitution? »(Paillat, 1983). 
Parlant de l'isolement en milieu professionnel, Paugam (2016) dit qu'il s'agit de la phase ultime d'un processus, le produit d'une accumulation d'échecs qui conduit à une forte marginalisation. N'ayant plus aucun espoir de s'en sortir véritablement, ces personnes marginalisées ont le sentiment qu'elles sont inutiles à la société et qu'elles ont perdu le sens de leur vie. Elles s'adonnent alors à l'alcoolisme et la drogue à la recherche de l'équilibre perdu. Les résultats de notre étude établissent un lien avec ce que dit cet auteur. En effet, une partie de nos sujets se sentant marginalisée, se plonge quotidiennement dans la consommation de koutoukou (boisson alcoolisée fabriquée localement). Par ailleurs, les patients de notre étude, dans leur très grande majorité ne s'estiment pas malades, aussi affirment-ils très souvent dans leurs propos : "je n'ai pas l'impression d'être malade, je ne sens la maladie nulle part, je sens plutôt qu'on me trouve inutile». Dans le cadre de la maladie chronique et de la vieillesse, ce type de discours met en évidence qu'on est bien en face d'une expérience vécue par la victime âgée comme une disqualification sociale. Parce que la maladie demeure dans l'imaginaire de celle-ci, et que, l'état de vieillesse est incriminé à la précarité au sens du processus d'affaiblissement des liens sociaux. L'analyse de nos résultats montre que cet affaiblissement ne débute pas avec les premiers symptômes de la maladie mais bien en avant. Cette analyse correspond à celle de Paugam (1991). Les recherches de ce dernier lui ont permis de constater que le processus de disqualification sociale ne commence pas obligatoirement par l'expérience du chômage, mais que l'on peut trouver dans le monde du travail des situations de précarité comparables à l'expérience du chômage, au sens de la crise identitaire et d'affaiblissement des liens sociaux. Son enquête auprès des pauvres dans la société française montre qu'il existe trois phases d'exclusion bien différentes : la fragilité, la dépendance, la rupture. Moins de la moitié de nos populations étudiées semble franchir ce processus. Nos résultats montrent que le diabète et le vieillissement les ont rendues vulnérables et dépendantes de leurs progénitures.

Alors, le fait d'habiter sous le toit d'un tiers dans un quartier précaire comme MICAO, donne à certains sujets âgés le sentiment d'être dévalorisé. Ce sentiment se retrouve chez $11 \%$ des enquêtés. Les travaux de Paugam (op.cit) affirment que les populations fragiles connaissent l'épreuve du déclassement social, le plus souvent de nature professionnelle et ne souhaitent pas être considérés comme des assistés. Pour cet auteur, les trois grands types d'épreuves, c'est-à-dire la fragilité, la dépendance et la rupture peuvent s'enchaîner de sorte que les fragilisés deviennent assistés ou que les assistés deviennent marginaux. Les assistés dans son étude, sont pris en charge par les services d'action sociale et, de ce fait, dépendent des aides que la collectivité a prévus pour les plus démunis. Cette dynamique dans laquelle l'individu tire de son groupe d'appartenance à la fois une identité positive, source de sa 
reconnaissance sociale, et une protection face aux aléas de la vie n'est pas le cas à MICAO, si bien que la totalité de nos enquêtés souhaite ardemment bénéficier de l'aide des autorités comme cela se fait ailleurs dans le monde.

Eu égard à la situation vécue par les personnes âgées dans cette commune, la contribution à l'amélioration de leurs conditions constitue un défi pour les collectivités territoriales de la Côte d'Ivoire. En effet, Anttonen et Sipilä (2003) fait observer que dans tous les pays, la responsabilité de la prise en charge sociale des personnes âgées dépendantes, des handicapés et des enfants en bas âge repose avant tout sur la sphère privée de la famille. Cet auteur indique par conséquent que toutes les autres solutions d'offre sociale apparaissent complémentaires à ce principe de base. Pour lui, les offres sociales issues des actions des secteurs formels et informels (la famille, les pouvoirs publics, les Organisations Non Gouvernementales (ONG) et autres œuvres de bienfaisance) apparaissent comme des appuis additionnels à l'intervention étatique en faveur de la population vulnérable. Dans cet ordre d'idée, notre étude montre que la crise militaro-politique qu'a connu le pays en septembre 2002 a occasionné des licenciements massifs des travailleurs dans les usines. De ce fait, les familles à même de prendre en charge les parents âgés, vivent dans un état de tension permanente et particulièrement de pauvreté aiguë. Il apparaît donc important selon les enquêtés que le gouvernement se penche sur les conditions de vie des populations vulnérables notamment des personnes âgées. Ils sollicitent l'appui de l'Etat et surtout des élus locaux à travers la création d'emplois pour les jeunes, le bénévolat pour les séniors et la réduction du transport en autobus de la SOTRA et d'impôt foncier pour les personnes âgées. Par ailleurs, il ressort également des entretiens avec nos enquêtés qu'au niveau de la maltraitance ou de l'isolement des personnes âgées, la mise en place d'une réglementation particulière pour leur protection au plan juridique s'avère nécessaire.

La contribution des pouvoirs publics sollicitée par les enquêtés est illustrée par l'exemple des pays scandinaves : "les services de prise en charge sociale sont largement disponibles dans les pays nordiques (à l'exception de l'Islande). Les classes moyennes et supérieures font partie des bénéficiaires des services publics, ce qui est lié au fait que l'ensemble du système de services tient compte des intérêts des femmes. Les municipalités ont la responsabilité de la production de services. Elles ont le pouvoir de lever des impôts tout en bénéficiant du soutien de l'État. » (Sipilä et al. 1997). Ces auteurs font comprendre que dans les pays scandinaves, les municipalités sont les principales responsables de l'organisation des services sociaux. L'État fixe le cadre législatif, mais il ne contrôle pas le détail des activités locales. Les autorités territoriales s'autogouvernent, dans le sens où leurs représentants sont élus et où elles disposent du droit de lever l'impôt. Elles sont responsables de l'organisation des politiques sociales et bénéficient des subventions de la 
part de l'État. Par conséquent, les municipalités sont bien plus que des régions administratives et la notion de commune indique qu'il existe un lien de solidarité entre les concitoyens, précise (Bernard, 1982). A propos, il propose une société à l'image des Cuiva. Car chez les Cuiva, selon lui, les gens âgés ne forment pas une catégorie sociale identifiable pouvant être distinguée du reste de la société. La société Cuiva ne crée pas d'âge de la vieillesse : "une fois sorti de l'enfance, l'individu demeure jusqu'à sa mort, confondu à l'ensemble des adultes. Il s'agit d'une société sans spécialisation, sans stratification, sans classes sociales, où il n'y a pas d'espace social particulier aux vieillards et il n'y a pas non plus d'activités sociales dont ils sont exclus. Au contraire, la société veut éviter la brisure et traite ses vieillards comme si leur vieillesse n'existait pas. » (Bernard op. cit)

Vivre dans une société pour tous les âges, est une recommandation des Nations Unies (ONU). Lors du sommet de Madrid (2002), l'ONU recommande dans son plan d'action international contre le vieillissement la création d'une société où les personnes âgées auraient tous les droits et où chaque gouvernement s'occuperait de ses propres vieux. Les experts onusiens rejoignent ainsi la société Cuiva en soutenant l'idée de création d'une société pour tous les âges. Cette idée a été perçue par certains dirigeants africains comme la consécration du modèle de société à l'africaine. C'est-à-dire, une société plus humaine qui exclue toute forme de marginalisation des personnes âgées.

Le système sociétal des Cuiva en faveur des sujets âgés et les résolutions de l'ONU sont superposables aux suggestions recueillies auprès des seniors dans notre étude. En effet, les aînés interviewés, conscients que l'Etat ne peut tout faire, demandent cependant au gouvernement de s'occuper du bien-être social des personnes vulnérables. En second lieu, ils suggèrent la rationalisation et l'objectivation des discours des élus. Concrètement, les enquêtés demandent aux autorités le passage d'une solidarité subjective à une solidarité objective fondée sur une réglementation qui protège leurs droits.

Enfin ils mettent en évidence le fait que dans les consciences collectives, lorsque les solidarités primaires sont défaillantes, les citoyens peuvent compter sur la puissance publique qui est une émanation de la solidarité nationale. Dans leurs suggestions, ils relèvent que l'aide mutuelle participe à la reconstruction permanente du lien social, et cette aide est mise à contribution lorsque le lien social est brisé à l'issue des circonstances exceptionnelles comme les conflits armés, la maladie et la vieillesse.

\section{Conclusion}

A l'issu de cette étude, il ressort que les rapports entre la structure familiale et la disqualification des personnes âgées diabétiques sont particulièrement affaiblies par les profondes mutations socioculturelles en 
cours en Côte d'Ivoire. La course effrénée à l'enrichissement et l'urbanisation galopante ont des retentissements graves sur la santé, la place et le vécu des personnes âgées dans la société abidjanaise. La majorité des personnes âgées ne bénéficient pas de pension de retraite, et la solidarité familiale qui sert de filet de sécurité en ce qu'elle constitue le gage d'épanouissement des aînés se désagrège. Et de plus en plus, le piétinement de la réconciliation nationale, la crise identitaire, et le chômage des jeunes ne permettent plus à la famille d'assurer son rôle d'antan, c'est-à-dire de veiller à la santé physique et psychosociale des parents âgés souffrant de maladie chronique. Cette situation aggrave les conditions de vie des personnes âgées à MICAO où elles sont confrontées à des problèmes multifactoriels d'ordre social, sanitaire, économique et culturel. Face à cela, elles développent des troubles de la communication avec l'entourage de type de disqualification, d'irritabilité, d'isolement, qui entraînent une incompréhension entre les aidants familiaux et la personne âgée diabétique. Les politiques gouvernementales qui devraient prendre davantage en considération les réalités de cette situation sont quasiinexistantes. Or, si les pays riches se sont développés avant de vieillir alors, c'est dès maintenant qu'il faille élaborer des solutions adaptées au contexte culturel ivoirien pour espérer un vrai développement du pays.

\section{References :}

1. ANKOTCHE Amos, BINAN Yves et al, (2009), Graves conséquences du coût financier du diabète sur sa prise en charge, en dehors des complications, en Afrique sub-saharienne : l'exemple de la Côted'Ivoire, in Médecine des Maladies Métaboliques, volume 3, issue 1, january, p. 100-105.

2. ANTTONEN Anneli et SIPILÄ Jorma, (2003), Les politiques de prise en charge des personnes âgées en Finlande. Quelles politiques en Europe ? éd. PUR, P. 227-247.

3. ARCAND Bernard, (1982), La construction culturelle de la vieillesse, Revue Anthropologie et sociétés, vol $6 \mathrm{n}^{\circ} 3, \mathrm{p} .7-23$.

4. BAROU Jacques, (2012), Les immigrés d'Afrique subsaharienne en Europe : une nouvelle diaspora ? Revue européenne des migrations internationales, 28, P. 147-167.

5. BAROU Jacques et GALLOU Rémi, (2011), Vieillir et mourir en Afrique ou en France ? Regards croisés de deux générations d'immigrés subsahariens, gérontologie et société, n³4, P.117-145.

6. BIRAMA Djan Diakité, DIARRA Tiéman, et TRAORÉ Sidy, (1993), Recours aux soins et consommation médicale dans le quartier de Bankoni.

7. CARADEC Vincent, (2004). Vieillir après la retraite. Approche sociologique du vieillissement, Paris : PUF, p. 240. 
8. CARADEC Vincent, (2001), Sociologie de la vieillesse et du vieillissement, Paris, Nathan, P.128.

9. DUTHE, Géraldine, LAURENT Raphaël et Pison, Gilles. (2009), Vivre et mourir après 60 ans en milieu rural africain, Isolement, recours aux soins et mortalité des personnes âgées à Mlomp, Paris, Institut national d'études démographiques, Document de travail, 160, p.36.

10. KOUAMÉ, Aka, (1990) Le vieillissement de la population en Afrique, Ottawa : Centre de Recherches pour le développement International, $46 \mathrm{p}$.

11. LAPLANTINE François, (1986), Anthropologie de la Maladie Étude Ethnologique des Systèmes de Représentations Étiologiques et Thérapeutiques dans la Société Occidentale Contemporaine.

12. LORIAUX Michel, (2001), Vieillir au Nord et au Sud : convergences ou divergences ? Jeunesses, vieillesses, démographies et sociétés, Actes de la $27^{\text {ème }}$ Chaire Quételet, Louvain-la-Neuve, 29 mai, et des

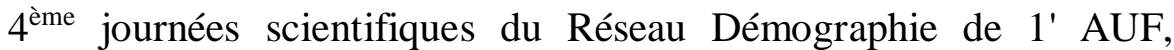
Louvain-la-Neuve, Academia-Bruylant, Paris, L'Harmattan, p. 25-43

13. LOUIS VINCENT Thomas, (1983), La vieillesse en Afrique noire, dans Communications, 37, P. 69-85.

14. LOUIS VINCENT Thomas, (1994), Vieillesse et mort en Afrique, in ATTIAS-DONFUT C., ROSENMAYR L., Vieillir en Afrique, Paris, PUF, p. 149-167.

15. NATIONS UNIES, (2001), Le vieillissement dans le monde. A la recherche d'une société pour tous les âges, New York, p. 122.

16. NEIRYNCK Isabelle et MELEN Marc, (2016), Deuxième conférence, Réorganisations familiales autour des maladies liées au vieillissement, http://www.systemique.be/spip consulté le 18 octobre 2018.

17. OMS, (2016), Rapport Mondial sur le Diabète.

18. PAILLAT Paul, (1983), Trop souvent seul, Gérontologie et société, 27.

19. PAUGAM Serge, (2016), La disqualification sociale, Essai sur la nouvelle pauvreté, Paris, PUF, P. 52-62

20. PHILIPPE Antoine, (2007), Les relations intergénérationnelles en Afrique : Approche plurielle, Ceped, collection Rencontres, Paris, p. 255.

21. POULAIN, Jean-Pierre, (1996), « L'alimentaire, un champ sociologique ? »; in Sociologie et anthropologie de l'alimentation, Portugal, 15ème colloque de 1'A.I.S.L.F Evora C.R, p.17.

22. POULAIN, Jean-Pierre et CORBEAU, J.P. (2002), Penser l'alimentation. Entre imaginaire et rationalité ; Paris, Editions Privat, p 209. 
23. ROSAY Hélène, (2004), Prise en charge des personnes âgées dans les sociétés traditionnelles, in Études sur la mort, vol.2, n¹26, p.27-36.

24. TOURE Abdou, (1984), Le «vieux » et «la vieilles»: Situation et rôle des personnes âgées en Côte d'Ivoire, in communication présentée au colloque de gérontologie sociale organisé par 'Université d'AixMarseille, France, p. 9-12.

25. TANOH Ahou Clémentine, (2007), les conditions de vie des personnes âgées en Côte d'ivoire : Regard sur la maltraitance à Adjamé village, P. 101. 